\title{
Action Learning Cohort Series: An Innovative Community-Engaged Approach for Translating Research Into Practice
}

\author{
Tiffany L. Young, $\mathrm{PhD}^{1}$ (iD \\ Lori Carter-Edwards, $\mathrm{PhD}, \mathrm{MPH}^{1}$ \\ Leah Frerichs, $\mathrm{PhD}^{1}$ \\ Melissa A. Green, $\mathrm{MPH}^{1}$ \\ Kristen Hassmiller-Lich, $\mathrm{PhD}^{1}$ \\ Elisa Quarles, $\mathrm{BA}^{1}$ \\ Gaurav Dave, MD, DrPH, $\mathrm{MPH}^{1}$ \\ Giselle Corbie-Smith, MD, MSc ${ }^{1}$
}

Cultivating strong partnerships among community and academic stakeholders expedites the translation of research findings into practice and communities by enhancing opportunities for research dissemination and implementation. However, the lack of systematic methods for community stakeholder engagement may decelerate the translational research process. The North Carolina Translational Research and Clinical Sciences Institute implemented an innovative approach to community engagement called the Action Learning Cohort (ALC) Series. The ALC Series, a workgroup extension of a professional conference, used action learning and systems thinking strategies to conceptualize and develop a product aimed at preventing, treating, and controlling hypertension in eastern North Carolina. We evaluated the acceptability and practicality of the ALC Series using survey, focus group, and interview pilot data. Action learning and systems thinking strategies led ALC stakeholders to develop and disseminate The Empathy Building Resource Guide: A Toolkit for Enhancing Patient-Provider Relationships in the Treatment, Management, and Prevention of Hypertension. Stakeholders rated the Series as satisfactory and acknowledged gains in knowledge and desire for engagement with fellow ALC stakeholders beyond the Series. The ALC Series approach is a potentially practical and acceptable model for systematically engaging community stakeholders in translating knowledge into a product that addresses health topics like hypertension.

Keywords: community-engaged research; translational research; systems thinking methods; action learning principles; empathy

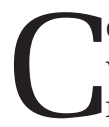
ommunity engagement is defined as a process of working with individuals in a collaborative, partnership, or coalition who are connected by

\footnotetext{
${ }^{1}$ University of North Carolina at Chapel Hill, Chapel Hill, NC, USA
}

Authors' Note: The authors wish to thank the Action Learning Cohort stakeholders, The Evidence Academy on Hypertension Steering Committee, Eastern North Carolina Health Organizations, UNC CTSA staff and leadership, and the American Heart Association who have made the ALC Series and dissemination of the Empathy Building Guide possible. The project described in the article was supported by the National Center for Advancing Translational Sciences (NCATS), National Institutes of Health, through Grant Award Number UL1TR002489. The content is solely the responsibility of the authors and does not necessarily represent the official views of the NIH. Address correspondence to Tiffany L. Young, The North Carolina Translational and Clinical Sciences (NC TraCS) Institute, University of North Carolina at Chapel Hill, Brinkhous-Bullitt Building, 2nd Floor CB 7064, 160 North Medical Drive, Chapel Hill, NC 27599-7064, USA; e-mail: lenell@email.unc.edu. 
geography, special interest, or circumstances to affect the lives of individual's in their community (CTSA Community Engagement Key Function Committee Task Force, 2011). Existing as a continuum, community engagement can range from simple outreach to shared leadership (CTSA Community Engagement Key Function Committee Task Force, 2011). Using community-engaged approaches, researchers have been able to increase the quality and relevance of research, enhance the practicality and validity of data collection and analysis efforts, and improve the dissemination and use of research results (Ahmed \& Palermo, 2010; Minkler, Salvatore, \& Chang, 2017). At various levels of engagement, academic and community partners have worked together to rally resources, influence systems and community relationships, and serve as catalyst for affecting health-related policy, programs, and practice (Ahmed \& Palermo, 2010).

The National Institutes of Health's Clinical and Translational Science Award Program promotes community engagement as a strategy to accelerate the translation of research into practice. While researchers have made recommendations to integrate community engagement at each stage of the translational research spectrum, community engagement is typically used during the T3 (translation to practice) and T4 (translation to population health) stages (Callard, Rose, \& Wykes, 2012; Treem, Schneider, Zender, \& Sorkin, 2018; Woolf, 2008). Strong partnerships among community and academic stakeholders increase the speed of translation by enhancing channels for dissemination and creating opportunities for implementing research findings (Cutforth \& Belansky, 2015). However, without appropriate and systematic methods for engagement, involvement in the translation process can be arduous and ineffective-leaving stakeholders feeling marginalized (Staley, 2009). As a result, knowledge and innovations may not reach intended users, and community relationships may be irreparably compromised, jeopardizing current and future opportunities for community-academic collaborations. Unfortunately, there is a paucity of literature describing systematic and effective methods for engaging community stakeholders in the translational research process.

One approach used to engage stakeholders in the translation of research findings is to hold a 1- or 2-day conference where health care professionals, researchers, and community members present model programs, innovations, and new research (Rohweder et al., 2016) on a specific topic like hypertension. These can be rich opportunities to stimulate new thinking about policies and practices (Louw \& Zuber-Skerritt, 2011). Inspired attendees often leave with intentions of taking action based on their new knowledge, but intentions seldom become reality (Haley, Wiessner, \& Robinson, 2009). Attendees need structured support to turn intentions into action; yet, conference organizers rarely create postconference opportunities that help attendees capitalize on new knowledge and readiness to apply new insights in community settings. Our team recognized this shortcoming and combined a one-day conference on hypertension in eastern North Carolina (NC) with an innovative, structured follow-up program called the Action Learning Cohort (ALC) Series (Rohweder et al., 2016). Here, we present an overview of the ALC processes and product: The Empathy Building Resource Guide; and results from our evaluation of the ALC as a community-engaged model in translational research.

\section{METHOD}

\section{Setting}

It is estimated that $35 \%$ of North Carolinians have been diagnosed with hypertension. At nearly $40 \%$, the eastern region of $\mathrm{NC}$ has one of the highest prevalence of hypertension in the state and is known as the "buckle" of the southeast "stroke belt." Furthermore, eastern NC has well documented racial disparities in hypertension (North Carolina Division of Public Health, 2017) associated with a host of biological, social, and environmental factors (Mozaffarian et al., 2016). To address the complexities of hypertension prevention, treatment, and management, The University of North Carolina (UNC) Clinical Science and Translational Institute organized a 1-day conference, the Evidence Academy on Hypertension (EA-HTN) in December 2014. Attended by 110 individuals who represented 20 NC counties, the conference was designed to present recent evidence in preventing, treating, and controlling hypertension. The conference encouraged co-learning experiences to promote and emphasize new evidence for improving health care systems and organizations within communities. Model programs and other infor-mation were presented by and among a variety of indi-viduals (i.e., health care professionals, researchers, and community members) who shared common interests in improving hypertension in eastern NC (Rohweder et al., 2016).

The ALC Series was implemented in the following Spring as a work group extension of the EA-HTN with the goal of developing a product aimed to address hypertension in eastern NC. To guide their work, the ALC used recommendations from a roundtable session at the conference that suggested the product broadly 
focus on enhancing partnerships to improve hypertension prevention, treatment, and control. Our use of action learning and systems thinking approaches during the Series helped further clarify the direction of the product.

\section{Stakeholder Recruitment}

Eligibility for the ALC included attendance at the EA-HTN conference and employment with an eastern NC organization that addressed hypertension. All attendees from the EA-HTN conference were sent email invitations to participate in the ALC. Aligned with action learning recommendations to form a small group to maximize effective interaction, communication, and problem solving (Marquardt, 2011), we aimed to recruit five to eight individuals for the Series. Once the e-mail invitations were sent, the first 10 interested respondents were chosen as ALC Series stakeholders. Ten stakeholders were selected to account for potential attrition.

\section{ALC Implementation}

Prior to implementing the ALC, the UNC team engaged in strategic planning that included curriculum and evaluation design as well as deciding meeting logistics such as selecting presenters and video conferencing and document storage tools. Because of our planning efforts, we were able to provide systematic processes, tools, and a collaborative environment for translating knowledge obtained from the conference into a tangible community-based product for addressing hypertension in eastern NC. Figure 1 provides an overview of ALC implementation process. The ALC Series implementation and its evaluation was approved by the UNC Institutional Review Board.

The ALC consisted of four 3-hour sessions. The initial session was in-person in eastern $\mathrm{NC}$ and the remaining three sessions were video-conferenced. Each session included a presentation by an invited speaker (e.g., American Heart Association), structured activities based on action learning principles and systems thinking methods to facilitate discussion and decision making, and assignments to help stakeholders translate their new knowledge into practice. Stakeholders discussed the presentations and assignments in 30-minute follow-up conference calls and identified ways to translate the information into actionable new practices.

Action learning framework. Action learning is a structured form of engagement in which individuals learn and work together to solve problems; it is used in a variety of contexts to develop leaders, build teams, and improve organizational capacity (Marquardt, 2011). Action learning principles include assembling a team, coaching, iteratively defining the problem, identifying questions, learning, taking action, and evaluating results.

In keeping with action learning principles (see Figure 1), our diverse team of stakeholders served as committed change agents. UNC staff and faculty served as coaches and facilitated the ALC sessions. Together, participants asked questions, exchanged knowledge, and engaged in problem solving to define and outline the details of their desired action. Topic experts presented on team science, systems thinking methods, and the American Heart Association's community initiatives. Stakeholders took action by using knowledge learned and shared during sessions to develop a product focused on addressing hypertension in their community. They evaluated the product and provided recommendations for disseminating it to health care providers.

Systems thinking methods. Systems thinking is an approach to problem solving that recognizes the importance of understanding the interrelated parts of a system and how the parts form an interdependent whole (Frerichs, Lich, Dave, \& Corbie-Smith, 2016). It fosters holistic, nonlinear thinking to explicate interrelated elements influencing behavior in a complex system (Frerichs et al., 2016). We used systems thinking to understand complexities of hypertension prevention, treatment, and management in the context of interrelated dynamic organizational, social, environmental, and biological factors. Specifically, we used a systems thinking tool system support mapping (SSM) and additional group facilitation to help the ALC identify and select a leverage point to target action.

Adapted from care mapping strategies (Antonelli \& Lind, 2012), SSM is a stakeholder-centered process that helps individuals depict their role(s) in their organizational and community systems. In our case, we instructed stakeholders to construct concentric circles on a large paper map that identified and described their: organizational/community role, specific responsibilities, needs/barriers and assets/resources associated with each responsibility, and wishes for being better supported in their work. We then used a nominal group process to allow stakeholders to discuss and prioritize areas of commonality among their SSMs, from which 10 potential focus areas for action emerged.

The ALC ranked the 10 focus areas and growing empathy within stakeholder groups emerged as their top choice. Finally, we used an impact matrix to identify and connect the types of stakeholders (health care 


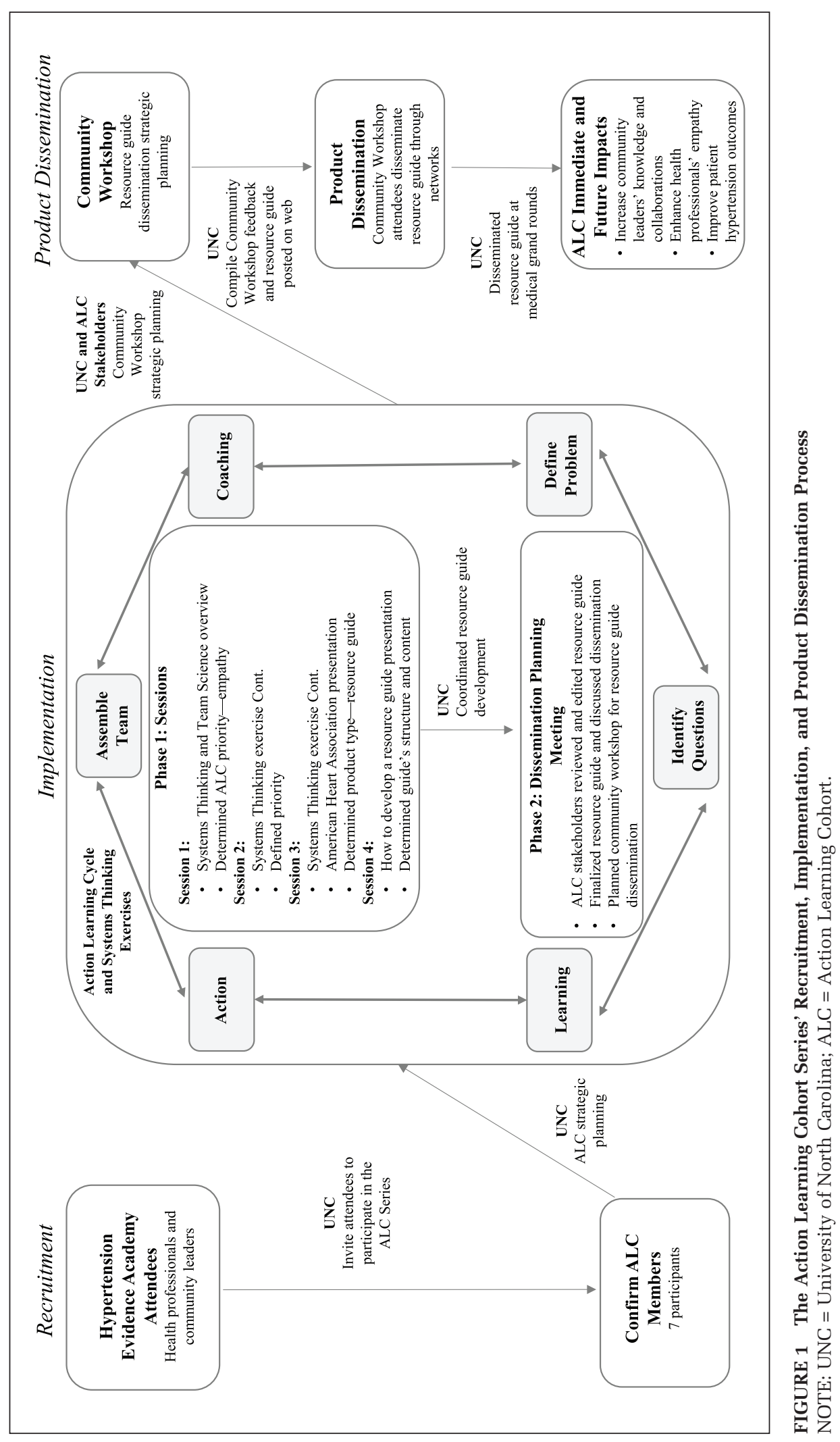


providers, community organization staff, and academic researchers) to the functions of HTN prevention, treatment, and management (e.g., self-management, tobacco use, health policies, etc.) they addressed. From the matrix, the stakeholders identified that health care providers were involved in the most functions that needed greater empathy. Specifically, they determined providers needed to enhance their empathic skills to effectively communicate with patients in general, but also to better understand and relay hypertension self-management strategies.

ALC stakeholders decided the best vehicle for educating health care providers about empathy was a resource guide of empathy-building strategies and tools. For more than 12 months, UNC staff supported the ALC by conducting literature reviews to find existing resources focused on enhancing providers' empathy skills when communicating with patients and drafted the guide's content with direction from ALC stakeholders.

\section{ALC Evaluation}

Measures and Data Collection. In this multimethod descriptive study, data collection methods included surveys, a focus group, and individual interviews. Completed after each session, we assessed practicality and acceptability with four questions regarding presentations, six questions about the exercises, and one question assessing overall satisfaction using a 4-point Likert-type scale. In addition, approximately 4 weeks after the final session, we conducted one focus group and individual interviews with stakeholders to gather their perspectives about session content, their learning experiences, and recommendations for ALC improvements. Focus group discussions and individual interviews were recorded for transcription. We collected all data during the spring and summer of 2015.

Analysis. In early 2016, our team analyzed survey data in SPSS version 24 (2016) using descriptive statistics. We transcribed focus group and interview data verbatim and exported all qualitative data into Atlas.ti version 7.5.6 (2014) for coding and thematic analysis. We reviewed all transcripts to create and define codes. Two researchers then separately coded the data and later reconciled any coding differences with a third researcher. Finally, we identified and analyzed themes that emerged from the data.

\section{ALC Product Dissemination Planning}

The North Carolina Translational and Clinical Sciences Institute partnered with the Area Health Education Centers to host a community workshop held in eastern NC in August 2017. With advertisement material provided by UNC, the ALC stakeholders invited professionals in their networks to participate in the workshop. During the workshop, the UNC team conducted a presentation on the EA-HTN and ALC Series, unveiled The Empathy Building Resource Guide, and partnered with workshop participants to codevelop a dissemination plan for the resource guide. To create the dissemination plan, UNC facilitated a brainstorming activity in which they divided the stakeholders into 4 groups and asked them the "who, what, when, and where" related to disseminating the guide. We used the feedback from the activity to further engage the stakeholders in a discussion on dissemination strategies and existing channels specific to eastern NC. See Figure 1 for the dissemination overview.

\section{RESULTS}

\section{Stakeholders}

The ALC Series included 10 participants. However, prior to the first session, three stakeholders dropped out and cited reasons such as new obligations and schedule changes. The final group consisted of seven stakeholders who represented community (program coordinators; $n=2$ ), faith-based (director and regional coordinator; $n=2$ ), academic (academician; $n=1$ ), and county health department (active nurse and retired/ volunteer nurse; $n=2$ ) organizations from eastern NC. Five stakeholders participated in the system support mapping exercise and all stakeholders participated in the nominal group process and ranking activities.

\section{ALC Series Practicality and Acceptability Evaluation}

Survey Results. Over the course of the ALC Series, stakeholders completed 19 postsession surveys (every stakeholder did not attend each session). Average ratings for survey questions are in Table 1. Overall, stakeholders were satisfied with the sessions; agreed session presentations and exercises were clear, organized, and presented at the right pace; and that they would be able to incorporate skills and information learned into their work.

Focus Group and Individual Interview Results. Five ALC stakeholders participated in a focus group. Two stakeholders who were unable to participate in the focus group due to their schedules participated in individual interviews. Four themes emerged from the qualitative data analysis: (1) gaining new knowledge and 
TABLE 1

Action Learning Cohort Series Evaluation Summary $(n=7)$

\begin{tabular}{|c|c|}
\hline Survey Item & Average Rating ${ }^{a}$ \\
\hline \multicolumn{2}{|l|}{ Presentations ${ }^{\mathrm{b}}$} \\
\hline Material was clear and organized & 3.2 \\
\hline Material was presented at the right pace & 3.1 \\
\hline Sufficient time for interaction, questions, and discussion among participants & 3.5 \\
\hline Able to apply what I learned in the presentation to my own practice/work activities & 3.3 \\
\hline \multicolumn{2}{|l|}{ Exercises $^{\mathrm{b}}$} \\
\hline Presenter demonstrated a good understanding of the material related to the exercise & 3.3 \\
\hline Directions for the exercise was clear and organized & 3.5 \\
\hline Exercise implemented at the right pace & 3.1 \\
\hline Sufficient time for interaction, questions, and discussion among participants & 3.2 \\
\hline Able to apply what I learned during the exercise to my own practice/work activities & 3.1 \\
\hline Gained new skills as a result of participating in the exercise & 3.1 \\
\hline \multicolumn{2}{|l|}{ Overall Sessions $^{\mathrm{c}}$} \\
\hline Overall level of satisfaction with the sessions & 3.3 \\
\hline
\end{tabular}

${ }^{a}$ Averages are the mean of the means across the four Action Learning Cohort sessions. The number of participants who completed the surveys varied across sessions. ${ }^{b}$ Level of agreement ranged from 1 strongly disagree to 4 strongly agree. ${ }^{c}$ Level of satisfaction ranged from 1 very satisfied to 4 very dissatisfied.

experiences, (2) ALC environment, (3) acceptability of the ALC sessions, and (4) wishes for future engagement.

Theme 1: Gaining new knowledge and experiences. Members of the ALC series were introduced to new material and information from the session facilitators as well as learning from others during the group sessions. Stakeholder made comments such as,

I think there [was] a lot of really good information ... the information about empathy was very helpful.

The stakeholders were also specific about particular resources they learned about during the series. One stakeholder discussed how they became more knowledgeable about The American Heart Association's resources. The joint learning effort allowed diverse ideas and goals to be discussed and expounded on. Specifically, one stakeholder commented,

. . . [We] got a lot of good opportunities [to] exchange ideas and learn about each other.

Theme 2: ALC environment. The Series' environment played a significant role in the stakeholders' opportunity to exchange ideas among one other and the facilitators. The stakeholders discussed their preference for in-person sessions versus video conferencing. For example, stakeholders commented,

I do want to say that I think having the first session in person was really important.

and

... just having face to face contact and like putting faces with names during the rest of the phone call conversations makes a lot more sense for me. So, I think that piece was really important.

Others commented on how the lack of in-person meetings hindered the collaborative process. Stakeholders also discussed how the ALC's learning environment was supportive. One stakeholder commented,

I agree with the person that said earlier it was a supportive enough environment . . . if you were clueless as to what you're doing, you could ask a question without feeling like you are stupid . . . 
Theme 3: Acceptability of ALC sessions. Each stakeholder expressed their level of satisfaction or dissatisfaction with the Series. Many stakeholders discussed benefits that made their experience pleasurable. However, some members discussed they were dissatisfied that the toolkit was not complete at Session 4,

... I was a little disappointed we did not move forward and get something developed or choose from what's out there and come up with the product...

Theme 4: Wishes for future engagement. Some members expressed expectations for future collaboration for the group. For example, one stakeholder commented,

I'm hoping this conversation will continuously grow into a continuous partnership, because that [would] definitely benefit our organization.

Most members made comments on improvements needed for the Series. Stakeholders discussed how visual aids could assist individuals with being more engaged in the sessions. One participated stated,

... if there was something visual . . it could always help you think of some resource or something that you could check on and get more of information.

\section{ALC Product}

ALC stakeholders concluded that greater empathy in provider interactions with patients could potentially improve hypertension outcomes through providers' heightened understanding of how patients' perspectives and complex lives influence hypertension selfmanagement and medication adherence. They developed The Empathy Building Resource Guide: A Toolkit for Enhancing Patient Provider Relationships in the Treatment, Management, and Prevention of Hypertension. The guide included an introduction, a description of empathy and the provision of care, how to use the resource guide, and additional resources. The Empathy Building Resource Guide is available for download at https://racs.unc.edu/index.php/services /engagement/empathy-building-resource-guide

\section{ALC Product Dissemination}

Twenty-three stakeholders, who largely represented health care $(44 \%)$, academic $(16.7 \%)$, and state organizations $(11 \%)$ attended the community workshop. Most of the attendees labeled themselves as health care professionals $(66.7 \%)$, with an average of 23.7 years of experience. As a result of the brainstorming activities, we decided to work with NC Area Health Education Centers to disseminate the guide at physician medical grand rounds. We have disseminated the guide at medical grand rounds in two local hospitals in eastern NC. We also disseminated the guide during a regional, medical grand round in the Summer of 2017. The 23 workshop stakeholders also disseminated the guide through their professional networks. We are currently working with NC Area Health Education Centers to extend dissemination to practice-based research networks throughout eastern NC.

\section{DISCUSSION}

As hallmark approaches of the ALC Series, the action learning framework and system thinking methods fostered the inspiration and development of The Empathy Resource Building Guide. The ALC stakeholders favorably rated the Series and acknowledged its satisfying and challenging aspects. Furthermore, the stakeholders were able to disseminate the guide through their professional networks and at medical grand rounds in eastern NC.

Action learning has been used in health care and public health settings, and through participants' engagement with one another, it has increased their knowledge in a given area and increased their professional networks (Bazos et al., 2013; Leggat, Balding, \& Schiftan, 2015). Action Learning has also been shown to enhance the process of knowledge coproduction (Lehmann \& Gilson, 2015), and facilitate organizational systemic change (Bazos et al., 2013). In our use of action learning, stakeholders gained knowledge and were able to expand their networks, which enhanced the stakeholders' ability to develop and disseminate a product that was relevant to eastern NC. Our hope is eventually the strategies in the Empathy Guide will diffuse into health care practice and facilitate positive systemic change in the prevention, control, and treatment of hypertension.

System thinking is an emerging area in public health (Leischow \& Milstein, 2006; Trochim, Cabrera, Milstein, Gallagher, \& Leischow, 2006) and has been used to help community stakeholders holistically understand behaviors affecting health and generate solutions that take advantage of community assets (Brennan, Sabounchi, Kemner, \& Hovmand, 2015). In addition to increasing knowledge and identifying solutions and areas that can be optimized for change, system thinking methods have also unveiled opportunities for stakeholders to disseminate 
identified solutions (Frerichs, Hassmiller Lich et al., 2018; Frerichs, Young, et al., 2018). Using system thinking methods, the ALC stakeholders were able to examine the context of advocating for hypertension in eastern NC from the varied perspectives of their fellow stakeholders. The contextual understanding allowed them to identify a nuanced construct like empathy as a solution to address hypertension and health care providers across the continuum of care as a target for dissemination.

Due to the evaluation findings, we recommend future ALCs should have more centrally located in-person sessions, accelerated decision making and action processes, and travel stipends for stakeholders who need it. While the stakeholders are responsible for the direction and structure of prioritized actions, an adequately staffed facilitator team is essential to coordinate efforts and compile information. Stakeholder gains in knowledge, skills, and willingness to use the new information in their organizational roles are a tangible demonstration of increased professional capacity within the community. Creating a postconference forum to capitalize on conference knowledge and networks is a worthy investment and should be considered a standard conference feature to enhance the dissemination and implementation of information.

The National Academy of Medicine indicates community-engaged models are integral to translate research to practice (Liverman, Schultz, Terry, \& Leshner, 2013). However, there are few models that systematically outline the process, describe specific techniques, and produce a specific product to address a health issue of concern to the community. Our ALC approach to community engagement clearly demonstrates the direct application of knowledge translation through action learning and systems thinking to create a product that addresses a persistent health issue. As such, the ALC Series model is an important contribution to translational research.

\section{Limitations}

Our work is limited because we have not assessed the long-term impacts of the ALC experience on stakeholders' work and their organizations. We detail one instance of the Series; thus, the series should be replicated additional times to validate its utility and feasibility. While our sample size was constrained by action learning guidelines, our process could have benefited from the perspectives of other types of stakeholders such as physicians, patients, family caregivers, and so on. Also, we implemented the ALC in the limited context of hypertension research and practice in a single region of
NC. However, we believe the approach is sufficiently flexible to be implemented in other contexts.

\section{Conclusion}

Initial evidence from this pilot suggests the ALC approach is a potentially practical and acceptable model for systematically engaging community stakeholders in translating knowledge into a product that addresses a specific topic such as hypertension. To further validate the Series and its impact, our next steps are to determine the acceptability and efficacy of The Empathy Building Resource Guide; assess longterm impacts of the ALC experience on stakeholder engagement in hypertension prevention, treatment, and management; develop and disseminate a manual consisting of curriculum, evaluation measures, and logistical considerations to ensure future replication; and implement the model in other contexts to further test its applicability. Furthermore, future research should include testing The Empathy Building Resource Guide for its ability to enhance health care provider's empathic skills to effectively work with patients to prevent, treat, and control their hypertension.

\section{Implications and Recommendations for Practice}

The ALC Series provides a novel, feasible process to help public health conference participants translate new knowledge on the latest research evidence into practice. It is an effective model for engaging diverse professional stakeholders in activities that involve structured tools and processes to improve collaborative and strategic decision making. With appropriate preparation, conference planners and researchers should consider using similar processes like our ALC Series as a community engaged, systematic method to accelerate the translational research process. Our experience with the ALC Series indicated that using the model can help enhance community-academic relationships beyond informal networking at conferences.

\section{ORCID iD}

Tiffany L. Young (iD https://orcid.org/0000-0003-1996-2624

\section{REFERENCES}

Ahmed, S. M., \& Palermo, A.-G. S. (2010). Community engagement in research: Frameworks for education and peer review. American Journal of Public Health, 100, 1380-1387.

Antonelli, R., \& Lind, C. (2012, October). Care mapping: An innovative tool and process to support family-centered, comprehensive care coordination. Paper presented at the Primary Care Innovation 
Conference of the Harvard Medical School Primary Care Center, Boston, MA.

Bazos, D. A., Schifferdecker, K. E., Fedrizzi, R., Hoebeke, J., Ruggles, L., \& Goldsberry, Y. (2013). Action-learning collaboratives as a platform for community-based participatory research to advance obesity prevention. Journal of Health Care for the Poor and Underserved, 24(2), 61-79.

Brennan, L. K., Sabounchi, N. S., Kemner, A. L., \& Hovmand, P. (2015). Systems thinking in 49 communities related to healthy eating, active living, and childhood obesity. Journal of Public Health Management and Practice, 21(Suppl. 3), S55-S69. doi:10.1097/ phh.0000000000000248

Callard, F., Rose, D., \& Wykes, T. (2012). Close to the bench as well as at the bedside: Involving service users in all phases of translational research. Health Expectations, 15, 389-400. doi:10.1111/j.1369-7625.2011.00681.x

CTSA Community Engagement Key Function Committee Task Force. (2011). Principles of Community Engagement (2nd ed.). Washington, DC: National Institutes of Health.

Cutforth, N., \& Belansky, E. S. (2015). A community-engaged approach to translating research into practice: A physical education story. Progress in Community Health Partnerships, 9, 571582. doi:10.1353/cpr.2015.0082

Frerichs, L., Hassmiller Lich, K., Young, T. L., Dave, G., Stith, D., \& Corbie-Smith, G. (2018). Development of a systems science curriculum to engage rural African American teens in understanding and addressing childhood obesity prevention. Health Education \& Behavior, 45, 423-434. doi:10.1177/109019 8117726570

Frerichs, L., Lich, K. H., Dave, G., \& Corbie-Smith, G. (2016). Integrating systems science and community-based participatory research to achieve health equity. American Journal of Public Health, 106, 215-222. doi:10.2105/AJPH.2015.302944

Frerichs, L., Young, T. L., Dave, G., Stith, D., Corbie-Smith, G., \& Hassmiller Lich, K. (2018). Mind maps and network analysis to evaluate conceptualization of complex issues: A case example evaluating systems science workshops for childhood obesity prevention. Evaluation and Program Planning, 68, 135-147. doi:10.1016/j.evalprogplan.2018.03.003

Haley, K. J., Wiessner, C. A., \& Robinson, E. E. (2009). Encountering new information and perspectives: Constructing knowledge in conference contexts. Journal of Continuing Higher Education, 57(2), 72. doi:10.1080/07377360902964384

Leggat, S. G., Balding, C., \& Schiftan, D. (2015). Developing clinical leaders: The impact of an action learning mentoring programme for advanced practice nurses. Journal of Clinical Nursing, 24, 1576-1584.

Lehmann, U., \& Gilson, L. (2015). Action learning for health system governance: The reward and challenge of co-production. Health Policy and Planning, 30, 957-963. doi:10.1093/heapol/czu097
Leischow, S. J., \& Milstein, B. (2006). Systems thinking and modeling for public health practice. American Journal of Public Health, 96, 403-405. doi:10.2105/AJPH.2005.082842

Liverman, C. T., Schultz, A. M., Terry, S. F., \& Leshner, A. I. (2013). The CTSA program at NIH: Opportunities for advancing clinical and translational research. Washington, DC: National Academies Press.

Louw, I., \& Zuber-Skerritt, O. (2011). The learning conference: Knowledge creation through participation and publication. Learning Organization, 18, 288-300. doi:10.1108/09696471111132504

Marquardt, M. J. (2011). Optimizing the power of action learning: Real-time strategies for developing leaders, building teams and transforming organizations (2nd ed.). Boston, MA: Nicholas Brealey.

Minkler, M., Salvatore, A., \& Chang, C. (2017). Participatory approaches for study design and analysis in dissemination and implementation research. In R. C. Brownson, C. A. Graham, \& E. Proctor (Eds.), Dissemination and implementation research in health: Translating science to practice (2nd ed., pp. 175-190). New York, NY: Oxford University Press.

Mozaffarian, D., Benjamin, E. J., Go, A. S., Arnett, D. K., Blaha, M. J., Cushman, M., . . . Turner, M. B. (2016). Executive summary: Heart disease and stroke statistics-2016 update: A report from the American Heart Association. Circulation, 133, 447-454. doi:10.1161/cir.0000000000000366

North Carolina Division of Public Health. (2017). High blood pressure in North Carolina. Retrieved from http://www.communi tyclinicalconnections.com/_downloads/CCCPH_FactSheet _HighBloodPressure_FINAL_Jan2017.pdf

Rohweder, C. L., Laping, J. L., Diehl, S. J., Moore, A. A., Isler, M. R., Scott, J. E., . . . Corbie-Smith, G. (2016). Bridging research, practice, and policy: The "Evidence Academy" conference model. Journal of Public Health Management and Practice, 22, 200-203.

Staley, K. (2009). Exploring impact: Public involvement in NHS, public health and social care research. Retrieved from http:// www.invo.org.uk/wp-content/uploads/2011/11/Involve _Exploring_Impactfinal28.10.09.pdf

Treem, J. W., Schneider, M., Zender, R. L., \& Sorkin, D. H. (2018). Exploring the potential role of community engagement in evaluating clinical and translational science grant proposals. Journal of Clinical and Translational Science, 2, 139-146. doi:10.1017/ cts.2018.311

Trochim, W. M., Cabrera, D. A., Milstein, B., Gallagher, R. S., \& Leischow, S. J. (2006). Practical challenges of systems thinking and modeling in public health. American Journal of Public Health, 96, 538-546.

Woolf, S. H. (2008). The meaning of translational research and why it matters. JAMA Journal of the American Medical Association, 299, 211-213. doi:10.1001/jama.2007.26 